\title{
A Cooperative Scheduling Algorithm for the Coexistence of Fixed Satellite Services and 5G Cellular Network
}

\author{
Francesco Guidolin $^{\dagger *}$ Maziar Nekovee* Leonardo Badia ${ }^{\dagger} \quad$ Michele Zorzi $^{\dagger}$ \\ $\dagger$ Dept. of Information Engineering, University of Padova, via Gradenigo 6/B, 35131 Padova, Italy \\ * Samsung R\&D Institute UK, Communications House, South Street, Staines-upon-Thames.Surrey TW18 4QE, UKs
}

\begin{abstract}
The increasing demand for higher data rates has accelerated research on the next generation of mobile cellular networks $(5 G)$. One of the key factors of $5 G$ is the use of a larger bandwidth allocated in the millimeter wave (mmWave) frequency spectrum. In particular, one of the candidate bands is the portion of spectrum between 17 and $30 \mathrm{GHz}$ that is currently used by other technologies such as fixed satellite services (FSS) and the cellular network backhaul. In this paper, we analyze the coexistence between mobile services and FSS considering the main characteristics of the mmWave spectrum recently investigated in the literature. Moreover, we present a novel cooperative scheduling algorithm based on a game theoretic framework that exploits the use of analog beamforming at the base stations (BS). Finally, we show that adopting this algorithm ensure that the system meets the regulatory recommendation concerning the interference level at the FSS and at the same time provides a good user spectral efficiency.
\end{abstract}

Keywords-Millimeter wave communication; cellular networks; radio spectrum management; satellite communication; beamforming.

\section{INTRODUCTION}

The increasing popularity of smart phones and mobile data devices has made mobile communication an indispensable part of life for billions of people, generating an unprecedented growth of fast connectivity demand [1]. The current generation cellular network, including LTE and LTE-Advanced, already adopts advanced technologies such as orthogonal frequencydivision multiplexing (OFDM) and multi-input multi-output (MIMO) systems leaving limited room to further improvements [2]. Moreover, the current spectrum between $700 \mathrm{MHz}$ and $2.6 \mathrm{GHz}$ is saturated and an enlargement of the current systems bandwidth seems impossible within this frequency range. The most interesting candidate to face these challenges is the development of a new generation cellular network deployed in the mmWave spectrum [3]. Recent studies have demonstrated that exploiting the small wavelength of mmWave frequencies, it is possible to use large antenna arrays and overcome the large pathloss perceived within this portion of the spectrum [4][5] making the use of these frequencies feasible.

The spectrum between 17 and $30 \mathrm{GHz}$ is one of the candidates for the deployment of the next generation cellular network. Currently, part of this band is allocated on a coprimary basis to fixed services (FSs) and fixed satellite services (FSS) [6]. The FSS uplink (from FSS to satellite) is allocated in the band from 27.5 to $30 \mathrm{GHz}$ and the downlink (from satellite to FSS) is allocated from 17.3 to $21 \mathrm{GHz}$ [7]. It is then important to study the coexistence between FSS and the cellular network to understand whether the mobile service operating within these frequencies may affect the functionalities of the satellite services. Similar investigations have been proposed by the international telecommunication union (ITU) for the spectrum sharing between FSS and IMTadvanced systems in the frequency band from 3.4 to $6.4 \mathrm{GHz}$ [8][9].

In this work we present a novel cooperative scheduling algorithm for cellular BSs that, exploiting a game theoretic framework, regulates the FSS-cellular BSs coexistence maintaining the interference over noise level $(I / N)$ at the FSS below the threshold indicated by the regulatory recommendations. In particular, we model the scenario as a potential game [10]. This technique has been applied recently in the literature to design several wireless network problems. For instance, a game theoretic solution based on potential games for joint channel selection and power allocation in cognitive radio networks is presented in [11]. Exploiting the potential game framework, a resource allocation scheme in a multicell OFDMA uplink scenario for energy-efficient power control is proposed in [12]. Moreover, a distributed potential game-based algorithm that addresses the minimum transmission broadcast problem in wireless networks is presented in [13].

Different from [14], where we study the effect of several system parameters on the performance of coexisting FSS and cellular systems, the aim of our work is to design a cooperative scheduling algorithm where each BS schedules one user considering the achievable spectral efficiency and the impact on the FSS $I / N$ level. We develop our framework in the FSS downlink band considering the results provided in the literature on the mmWave spectrum [15][16] and the ITU recommendations. Moreover, our potential game formulation ensures equilibrium of user scheduling. We present three different versions of the algorithm: the first is based on throughout maximization, the second is based on FSS interference minimization, and the third considers both aspects. Finally, we show how, applying the algorithm proposed, it is possible to meet the regulatory recommendations and at the same time to reach a high level of spectral efficiency.

The paper is organized as follows. Section II describes the 
model considered, focusing on the standard system parameters used in the analysis, and Section III presents the cooperative scheduling algorithm proposed. Section IV gives the performance evaluation for the different scenarios considered. Finally, conclusions are drawn and future works are discussed in Section IV.

Remark: Throughout the paper, we use boldface letters for vectors and matrices, and we denote with $(\cdot)^{T}$ the conjugate transpose.

\section{SySTEM MODEL}

We consider the scenario depicted in Fig. 1 that corresponds to the downlink band of the FSS system at $18 \mathrm{GHz}$ [14]. The cellular BSs are deployed in tiers around the FSS according to the BSs intersite distance $d_{i}$ and the protection distance $d_{p}$ that represents the distance between the FSS and the first tier of BSs. The users are distributed randomly within the BSs coverage area and each BS selects one user at a time. We assume that each BS and each UE are equipped with $N_{U E}$ and $N_{B S}$ antennas, respectively. We define as primary link the transmission from the satellite to the FSS while as secondary link the connection from a cellular BS to a mobile user equipment (UE). The FSS is subject to additional interference through the interfering links from the BSs to the FSS, given by the sum of all the interference generated by the BSs.

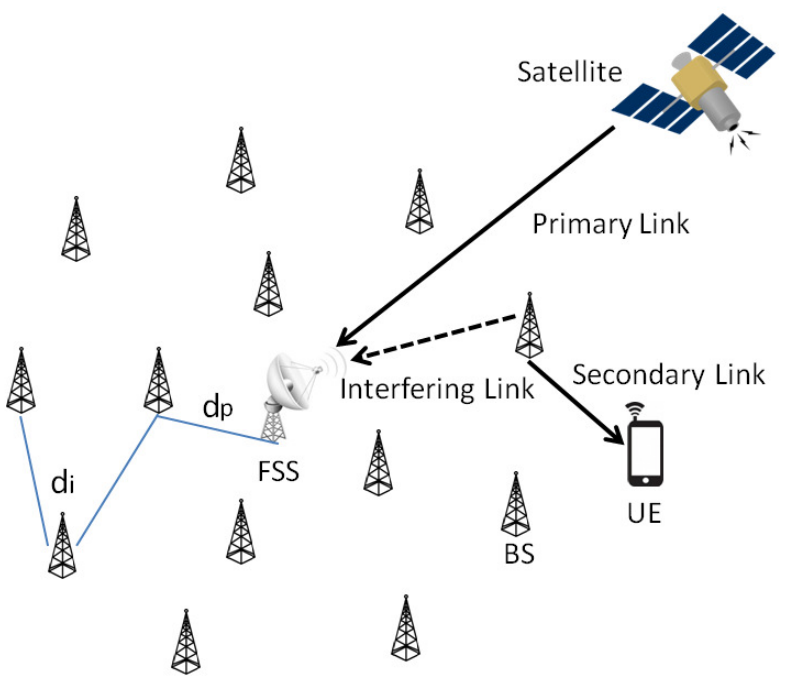

Fig. 1. FSS-BS coexistence scenario

The interference (on a log scale) generated by BS $b$ to the FSS can be computed as

$$
I_{b}=P_{B S}+G_{B S}+G_{F S S}(\phi)-L(d)
$$

where $P_{B S}$ is the BS transmission power, $G_{B S}$ is the BS antenna gain, $G_{F S S}(\phi)$ is the FSS antenna gain in the direction $\phi$, where $\phi$ is the angle between the main FSS antenna lobe and BS $b$, and $L(d)$ is the pathloss component at distance $d$ which in turn is the FSS-BS distance.

According to [17] we adopt a double-directional geometry based stochastic model with $L$ scatterers, where $L$ is limited to a small number for a mmWave scenario. The $N_{U E} \times N_{B S}$ channel matrix $\mathbf{H}$ can be computed as

$$
\mathbf{H}=\sqrt{\frac{N_{B S} N_{U E}}{L}} \sum_{\ell=1}^{L} \alpha_{\ell} \mathbf{a}_{U E}\left(\gamma_{\ell}^{U E}\right) \mathbf{a}_{B S}^{*}\left(\gamma_{\ell}^{B S}\right)
$$

where $\alpha_{\ell}$ is the complex gain of the $\ell^{t h}$ path and $\gamma_{\ell}^{U E}, \gamma_{\ell}^{B S} \in$ $[0,2 \pi]$ are uniformly distributed random variables that represent the angles of arrival and departure, respectively. Finally, $\mathbf{a}_{U E}$ and $\mathbf{a}_{B S}$ are the antenna array responses at the UEs and BSs, respectively. Assuming uniform linear arrays, $\mathbf{a}_{B S}$ can be written as

$\mathbf{a}_{B S}=\frac{1}{\sqrt{N_{B S}}}\left[1, e^{j \frac{2 \pi}{\lambda} D \sin \left(\gamma_{l}^{B S}\right)}, \ldots, e^{j\left(N_{B S}-1\right) \frac{2 \pi}{\lambda} D \sin \left(\gamma_{l}^{B S}\right)}\right]$

where $D$ is the distance between antennas. Similarly, $\mathbf{a}_{U E}$ can be computed by substituting $N_{B S}$ and $\gamma_{\ell}^{B S}$ in (3) with $N_{U E}$ and $\gamma_{\ell}^{U E}$, respectively.

The BS antenna gain in $\mathrm{dB}$ is

$$
G_{B S}=G_{o m n i}+G_{B F}
$$

where $G_{o m n i}$ is the conventional antenna gain when no beamforming techniques are applied. Conversely, $G_{B F}$ is the beamforming gain and depends on the beam configuration selected by the BS controlling phase and magnitude of the input signal to each antenna and on the channel coefficients. We assume that the BSs can select the beam configuration within a predefined beam codebook with cardinality $N_{t}$ that uniformly covers the azimuth directions. The codebooks at the transmitter and the receiver are formed by $N_{t}$ and $N_{r}$ weight vectors $\left\{\mathbf{v}_{1}, \ldots, \mathbf{v}_{N_{t}}\right\}\left\{\mathbf{w}_{1}, \ldots, \mathbf{w}_{N_{r}}\right\}$ of size $N_{B S}$ and $N_{U E}$, respectively. Each vector is computed as $\mathbf{v}_{i}=\mathbf{a}\left(\rho_{i}\right)$ and $\mathbf{w}_{k}=\mathbf{a}\left(\theta_{k}\right)$ where $\rho_{i}$ and $\theta_{k}$ are the azimuth angles for the $i$-th transmit RF beam and $k$-th receive RF beam. We assume a multiple-input-single-output (MISO) scenario, in which the beamforming gain in the direction of the FSS antenna is

$$
G_{B F}=10 \log \left(\left|\mathbf{v}_{i}^{T} \mathbf{h}_{F S S}\right|^{2}\right)
$$

where $\mathbf{v}_{i}$ is the beamforming precoding vector selected by the $\mathrm{BS}$ and $\mathbf{h}_{F S S}$ is the channel matrix between the BS and the FSS.

The FSS antenna gain is computed as a function of the offboresight angles, which can be calculated using the model in [18]. Considering $\vartheta$ as the azimuth of the BS w.r.t. the FSS $\mathrm{Rx}$ main lobe, the off-boresight angles $\phi$ of the BS towards the FSS can be calculated as

$$
\phi=\arccos (\cos (\alpha) \cos (\epsilon) \cos (\vartheta)+\sin (\alpha) \sin (\epsilon))
$$

where $\alpha$ is the FSS elevation angle and $\epsilon$ is computed as

$$
\epsilon=\frac{h_{t}-h_{s}}{d}-\frac{d}{2 r}
$$


where $h_{s}$ and $h_{t}$ are the heights of the BS and the FSS in meters, respectively, while $r$ is the effective Earth radius $(\approx$ $\left.8.5 \cdot 10^{3} \mathrm{~km}\right)$. The FSS off-boresight antenna gain pattern in $\mathrm{dB}$ can be computed as [19]

$$
G_{F S S}(\phi)=\left\{\begin{array}{cc}
G_{\max } & \text { if } 0^{\circ}<\phi<1^{\circ} \\
32-25 \log \phi & \text { if } 1^{\circ} \leq \phi<48^{\circ} \\
-10 & \text { if } 48^{\circ} \leq \phi \leq 180^{\circ}
\end{array}\right.
$$

where $G_{\max }$ is the main beam axis FSS antenna gain.

The level of interference allowable at the FSS is regulated by ITU. For the "short term" interference, recommendation [20] indicates that interference from fixed service systems should not cause the BER to exceed $10^{-4}$ for more than $0.03 \%$ of any month nor cause the BER to exceed $10^{-3}$ for $0.005 \%$ of any month. These interference allowances, in terms of percentage of system noise, can be converted into corresponding values of $I / N$, leading to -2.4 and $0 \mathrm{~dB}$, respectively. To evaluate the performance of our algorithm, we consider the regulatory recommendation for the "long term" interference that refers to a percentage of time greater than $20 \%$. In this case, recommendation [20] allows an interference that would give rise to a BER of $10^{-6}$. The value for $20 \%$ of the time is computed in [21] and is equal to $-10 \mathrm{~dB}$.

\section{COOPERATIVE SCHEDULING ALGORITHMS}

In this section we describe the cooperative scheduling algorithm proposed. The aim of this algorithm is to improve the BSs-FSS coexistence reducing the minimum $d_{p}$ required to satisfy the standard interference limit threshold at the FSS. The main idea is to coordinate the user transmissions in order to control the interference at the FSS and at the same time preserve the user average spectral efficiency. The interactions among the BSs can be modelled using a game theoretic framework. In particular, by modelling the scenario as an exact potential game, it is possible to ensure that a pure Nash equilibrium can be reached [10][11]. A characteristic of a potential game is that any unilateral change of utility, $U\left(s_{i}, s_{-i}\right)$, corresponds to a difference of a potential function, $F(s)$, for every player and for every choice of the other players. The potential function models the information associated with the improvement paths of a game instead of the exact utility of the game. Our scenario can be modelled in a normal form game $\Gamma=\left\{B,\left\{S_{i}\right\}_{i \in B},\left\{U_{i}\right\}_{i \in B}\right\}$, where each player corresponds to a $\mathrm{BS}, B$ is the set of players and therefore the number of BSs, and $S_{i}=\{1,2, \ldots, K\}$ is the set of strategies of player $i$. Considering that $K$ users are deployed within the coverage area of a player $i$, the strategy played by $i$ consists of the selection of a specific user within the $K$ deployed in its area. For every player $i$ in $\Gamma$, the utility function $U_{i}$ is a function of strategy $s_{i}$ selected by player $i$, and the strategies of the other players, globally denoted as $s_{-i}$.

In our algorithm we assume that the actions of the players are taken sequentially by randomly selecting one player in each algorithm iteration. The procedure terminates when the algorithm converges to a stable scheduling configuration. We also assume that each BS has a global knowledge of the system parameters that is exploited to optimize the utility function, and that the channel conditions are constant during each algorithm realization. When a BS is selected, the user that maximizes the BS's utility function is scheduled. We emphasize that, if the game considered corresponds to an exact potential game, the equilibrium convergence is guaranteed and the configuration of the users scheduled is stable. We define three different approaches based on three different utility functions: a maximum rate approach (MaxRate), a minimum interference approach (MinInt) and finally an algorithm based on the linear combination of the previous ones (LinComb).

The aim of the first algorithm is to maximize the mean spectral efficiency of the users considering within the utility function the signals received by the selected user and the intercell interference. In this case, we define the utility of player $i$ given a certain strategy $s_{i}$ as

$$
\begin{aligned}
U_{i}^{M R}\left(s_{i}, s_{-i}\right) & =p_{j i}\left|\mathbf{v}_{i}^{T} \mathbf{h}_{j i}\right|^{2}-\sum_{b=1, b \neq i}^{B} p_{j b}\left|\mathbf{v}_{b}^{T} \mathbf{h}_{j b}\right|^{2} \\
& -\sum_{m=1, m \neq j}^{M} p_{m i}\left|\mathbf{v}_{i}^{T} \mathbf{h}_{m i}\right|^{2}
\end{aligned}
$$

where $j$ is the user scheduled by BS $i$ when strategy $s_{i}$ is adopted and $p_{j i}$ is the power at user $j$ transmitted from $i$. The utility function consists of three terms. The first term represents the power received by the user scheduled by $i$, the second term indicates the inter-cell interference received by user $j$ and, the third term represents the interference generated by $i$ on the users scheduled by the other BSs.

Conversely, the aim of the MinInt algorithm is to minimize the FSS interference. In this case the utility function is:

$$
U_{i}^{M I}\left(s_{i}, s_{-i}\right)=-\xi(I / N)
$$

where $\xi(I / N)$ is a function of the interference generated by the BSs to the FSS. In particular $I / N$ is defined as:

$$
I / N=\sum_{b=1, b \neq i}^{B} I_{b}-N
$$

where $I_{b}$ is defined in (1) and $N$ is the noise level. The function $\xi(I / N)$ is designed in order to penalize the strategies that generate large interference at the FSS and to provide more flexibility to the users selection if the FSS interference is lower than the recommended threshold. $\xi(I / N)$ is set as

$$
\xi(I / N)=\left\{\begin{array}{cl}
0 & \text { if } I / N<I / N_{0} \\
\frac{I}{1-e^{-\left(I / N-I / N_{0}\right)}} & \text { if } I / N \geq I / N_{0}
\end{array}\right.
$$

where $I / N_{0}$ is fixed to a value lower than the recommended $I / N$ threshold, $-12 \mathrm{~dB}$, in order to control the interference at the FSS.

Finally, we define the LinComb algorithm where the effects on the users and on the FSS are considered together. In this 
TABLE I. MAIN SYSTEM PARAMETERS

\begin{tabular}{|c|c|}
\hline Parameter & Value \\
\hline Carrier frequency & $18 \mathrm{GHz}$ \\
\hline Total downlink bandwidth & $500 \mathrm{MHz}$ \\
\hline BS transmit power & $30 \mathrm{dBm}$ \\
\hline BS antenna height & $20 \mathrm{~m}$ \\
\hline BS omnidirectional antenna gain & $6 \mathrm{dBi}$ \\
\hline BSs intersite distance & $500 \mathrm{~m}$ \\
\hline BS inter-antenna distance & $\lambda / 2$ \\
\hline BS beam codebook cardinality & 16 \\
\hline BS number of antennas & 16 \\
\hline FSS antenna main lobe gain & $42.1 \mathrm{dBi}$ \\
\hline FSS antenna diameter & $2.4 \mathrm{~m}$ \\
\hline FSS antenna height & $2 \mathrm{~m}$ \\
\hline Elevation angle & $10^{\circ}$ \\
\hline Pathloss model & $61.39+10 \times 2.47 \mathrm{log}(d)[16]$ \\
\hline Number of scatterers & 3 \\
\hline Noise temperature & $300 \mathrm{~K}$ \\
\hline Number of users per BS & 10 \\
\hline Recommended $I / N$ level & $-10 \mathrm{~dB}$ \\
\hline
\end{tabular}

case, the utility function is

$$
\begin{aligned}
U_{i}\left(s_{i}, s_{-i}\right) & =p_{j i}\left|\mathbf{v}_{i}^{T} \mathbf{h}_{j i}\right|^{2}-\sum_{b=1, b \neq i}^{B} p_{j b}\left|\mathbf{v}_{b}^{T} \mathbf{h}_{j b}\right|^{2} \\
& -\sum_{m=1, m \neq j}^{M} p_{m i}\left|\mathbf{v}_{i}^{T} \mathbf{h}_{m i}\right|^{2}-\beta \xi(I / N)
\end{aligned}
$$

where $\beta$ is an adjusting parameter.

For all the algorithms it is possible to define an exact potential function that leads to a specific potential game. The proof is reported in the Appendix.

\section{Performance Evaluation}

In this section, we evaluate the performance reachable with the cooperative scheduling algorithm. In particular, we study the results achievable by the LinComb algorithm in terms of $I / N$ level at the FSS and of mean spectral efficiency of the users. We assume that the total downlink bandwith is $500 \mathrm{MHz}$ and the BSs allocate the power uniformly over this bandwith. The pathloss model is given exploiting the results presented in [16] on the mmWave band. Assuming a system effective noise temperature $\mathrm{T}$ equal to $300 \mathrm{~K}$, the one-sided noise power spectral density value results equal to $\mathrm{kT}=-143.82$ $\mathrm{dBW} / \mathrm{MHz}$, where $\mathrm{k}$ is the Boltzmann constant.

We assume that three tiers of BSs are deployed around the FSS and 10 user are randomly deployed within each BS coverage area. The number of antennas for each BS is fixed to 16. Considering the expected cell coverage in next generation cellular networks [22] we assume $d_{i}=500 \mathrm{~m}$. We emphasize that the markers reported in the graphs are used just to improve the curve visualization. The detailed system parameters are reported in Table I.

Fig. 2 shows the evolution of the $I / N$ level at the FSS for the different potential game algorithms proposed using the same configuration of users and starting from the same random set of scheduled users. As expected, the $I / N$ level for the

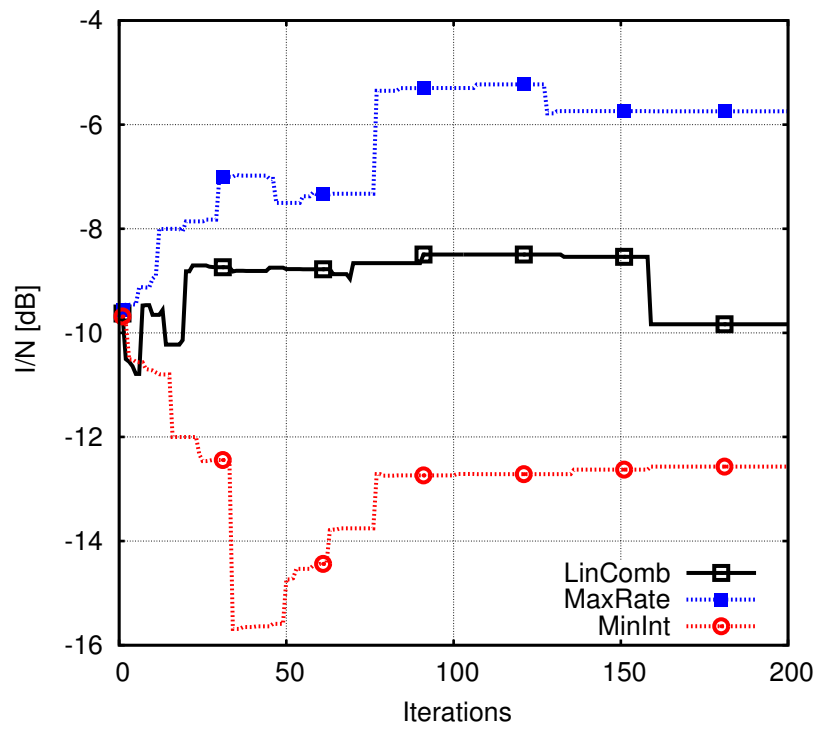

Fig. 2. $I / N$ evolution for the different algorithms considered

MinInt algorithm converges around $I_{0}$ since there is no reward for the BSs to schedule users that generate interference at the FSS. Conversely, the utility of the MaxRate algorithm is related just to the spectral efficiency of the users and the $I / N$ level converges to a higher value. Finally, the LinComb algorithm achieves an intermediate $I / N$ value. We emphasize that the converging values of the MinInt and LinComb algorithms can be modified selecting a different $I_{0}$ value.

Fig. 3 describes the CDF of the $I / N$ level at the FSS. The results have been obtained via Monte Carlo simulations using a different users configuration for each realization. As expected, the performance achieved by the MaxRate algorithm are above the limit imposed by regulations since there is no constraint on the interference at the FSS. Conversely, for the MinInt algorithm the $I / N$ level is maintained under the $-10 \mathrm{~dB}$ threshold recommended by the standard. Using the LinComb algorithm the standard threshold is achieved for almost $60 \%$ of the user configurations getting a significant improvement in comparison with the MaxRate case.

Fig. 4 shows the CDF of the user spectral efficiency $\nu$ for the different algorithms considered. As expected, the MinInt and MaxRate algorithms obtain the worst and the best results, respectively. Conversely, the PG algorithm achieves a result very close to the MaxRate algorithm even though, as depicted in Fig. 3, the interference at the FSS is maintained at low values. Thus, the LinComb algorithm provides a good tradeoff between guaranteeing a high user spectral efficiency and achieving an acceptable interference level at the FSS. Besides, by properly selecting the parameters $I_{0}$ and $\beta$, it is possible to regulate the BSs-FSS coexistence and to reduce the protection distance to increase the mmWave network coverage area.

\section{CONCLUSIONS AND FUtURE WORK}

In this paper, we investigated the coexistence feasibility of FSS and cellular BS in a mmWave scenario using a novel 


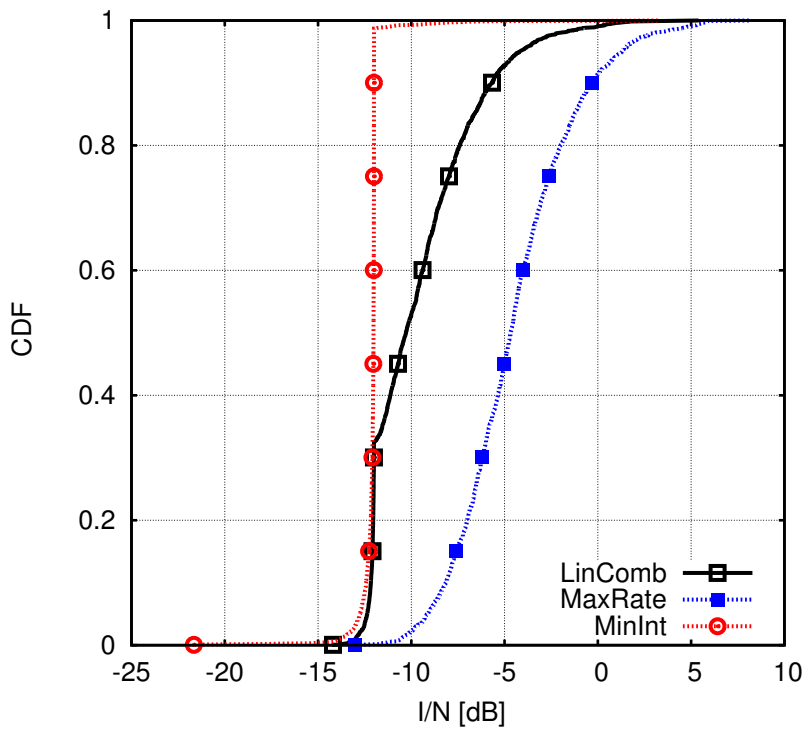

Fig. 3. $I / N \mathrm{CDF}$ for the different algorithms considered

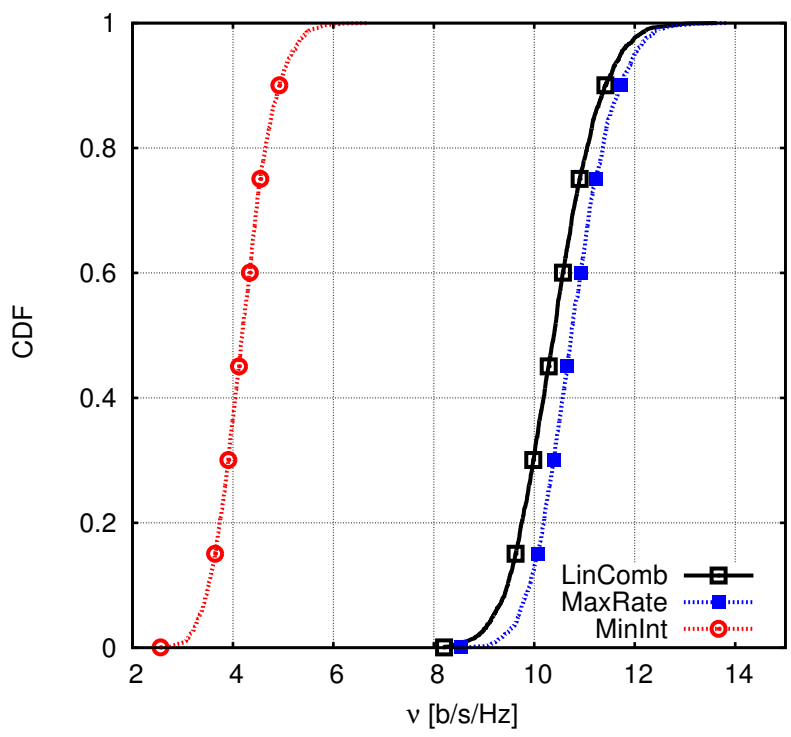

Fig. 4. $\nu \mathrm{CDF}$ for the different algorithms considered

cooperative scheduling algorithm based on a game theoretic framework. We showed that coordinating the BS scheduling and exploiting the characteristics of the mmWave spectrum, it is possible to meet the interference regulatory recommendations at the FSS and to achieve a good spectral efficiency at the users.

As future work, we intend to develop possible distributed cooperative algorithms of beamforming and scheduling among the BSs to mitigate the interference at the FSS and to reduce the required signalling among the BSs. Moreover, more complex scenarios such as heterogeneous networks and more realistic BS deployment could be considered.

\section{ACKNOWLEDGMENT}

We thank Profs. Barry Evans and Klaus Moessner from 5G Innovation Centre for valuable discussion and inputs.

\section{APPENDIX}

In order to show that the problems presented in Section III can be treated as potential games, we have to define a potential function for each scenario able to satisfy the propriety

$$
U_{i}\left(s_{i}, s_{-i}\right)-U_{i}\left(s_{i}^{\prime}, s_{-i}\right)=F\left(s_{i}, s_{-i}\right)-F\left(s_{i}^{\prime}, s_{-i}\right)
$$

Considering the set of BSs $\{1, \ldots, B\}$ and denoting as $q$ the user scheduled by BS $k$ with $k \in\{1, \ldots, B\}$, we can define $F(S)$ for the MaxRate algorithm as

$$
\begin{aligned}
F(S) & =F\left(s_{k}, s_{-k}\right) \\
& =\sum_{i=1}^{B}\left(p_{j i}\left|\mathbf{v}_{i}^{T} \mathbf{h}_{j i}\right|^{2}-\alpha \sum_{b=1, b \neq i}^{B} p_{j b}\left|\mathbf{v}_{b}^{T} \mathbf{h}_{j b}\right|^{2}\right. \\
& \left.-(1-\alpha) \sum_{m=1, m \neq j}^{M} p_{m i}\left|\mathbf{v}_{i}^{T} \mathbf{h}_{m i}\right|^{2}\right)
\end{aligned}
$$

with $0<\alpha<1$.

It is possible to isolate the terms depending on $s_{k}$ as

$$
\begin{aligned}
F(S) & = \\
& =p_{q k}\left|\mathbf{v}_{k}^{T} \mathbf{h}_{q k}\right|^{2}-\alpha \sum_{b=1, b \neq k}^{B} p_{q b}\left|\mathbf{v}_{b}^{T} \mathbf{h}_{q b}\right|^{2} \\
& -(1-\alpha) \sum_{m=1, m \neq q}^{M} p_{m k}\left|\mathbf{v}_{k}^{T} \mathbf{h}_{m k}\right|^{2} \\
& +\sum_{i=1, i \neq k}^{B}\left(p_{j i}\left|\mathbf{v}_{i}^{T} \mathbf{h}_{j i}\right|^{2}-\alpha \sum_{b=1, b \neq i}^{B} p_{j b}\left|\mathbf{v}_{b}^{T} \mathbf{h}_{j b}\right|^{2}\right. \\
& \left.-(1-\alpha) \sum_{m=1, m \neq j}^{M} p_{m i}\left|\mathbf{v}_{i}^{T} \mathbf{h}_{m i}\right|^{2}\right) \\
& =p_{q k}\left|\mathbf{v}_{k}^{T} \mathbf{h}_{q k}\right|^{2}-\alpha \sum_{b=1, b \neq k}^{B} p_{q b}\left|\mathbf{v}_{b}^{T} \mathbf{h}_{q b}\right|^{2} \\
& -(1-\alpha) \sum_{m=1, m \neq q}^{M} p_{m k}\left|\mathbf{v}_{k}^{T} \mathbf{h}_{m k}\right|^{2} \\
& +\sum_{i=1, i \neq k}^{B}\left(p_{j i}\left|\mathbf{v}_{i}^{T} \mathbf{h}_{j i}\right|^{2}-\alpha p_{j k}\left|\mathbf{v}_{k}^{T} \mathbf{h}_{j k}\right|^{2}\right. \\
& -\alpha \sum_{b=1, b \neq i, k}^{B} p_{j b}\left|\mathbf{v}_{b}^{T} \mathbf{h}_{j b}\right|^{2}-(1-\alpha) p_{q i}\left|\mathbf{v}_{i}^{T} \mathbf{h}_{q i}\right|^{2} \\
& \left.-(1-\alpha) \sum_{m=1, m \neq j, q}^{M} p_{m i}\left|\mathbf{v}_{i}^{T} \mathbf{h}_{m i}\right|^{2}\right)
\end{aligned}
$$


Let

$$
\begin{aligned}
Q\left(s_{-k}\right) & =\sum_{i=1, i \neq k}^{B}\left(p_{j i}\left|\mathbf{v}_{i}^{T} \mathbf{h}_{j i}\right|^{2}-\alpha \sum_{b=1, b \neq i, k}^{B} p_{j b}\left|\mathbf{v}_{b}^{T} \mathbf{h}_{j b}\right|^{2}\right. \\
& \left.-(1-\alpha) \sum_{m=1, m \neq j, q}^{M} p_{m i}\left|\mathbf{v}_{i}^{T} \mathbf{h}_{m i}\right|^{2}\right)
\end{aligned}
$$

Then

$$
\begin{aligned}
F(S) & =p_{q k}\left|\mathbf{v}_{k}^{T} \mathbf{h}_{q k}\right|^{2}-\alpha \sum_{b=1, b \neq k}^{B} p_{q b}\left|\mathbf{v}_{b}^{T} \mathbf{h}_{q b}\right|^{2} \\
& -(1-\alpha) \sum_{m=1, m \neq q}^{M} p_{m k}\left|\mathbf{v}_{k}^{T} \mathbf{h}_{m k}\right|^{2} \\
& -\sum_{i=1, i \neq k}^{B}\left(\alpha p_{j k}\left|\mathbf{v}_{k}^{T} \mathbf{h}_{j k}\right|^{2}+(1-\alpha) p_{q i}\left|\mathbf{v}_{i}^{T} \mathbf{h}_{q i}\right|^{2}\right) \\
& +Q\left(s_{-k}\right)-\beta \xi\left(I-I_{o}\right) \\
& =p_{q k}\left|\mathbf{v}_{k}^{T} \mathbf{h}_{q k}\right|^{2}-\sum_{b=1, b \neq k}^{B} p_{q b}\left|\mathbf{v}_{b}^{T} \mathbf{h}_{q b}\right|^{2} \\
& -\sum_{m=1, m \neq q}^{M} p_{m k}\left|\mathbf{v}_{k}^{T} \mathbf{h}_{m k}\right|^{2}+Q\left(s_{-k}\right)-\beta \xi\left(I-I_{o}\right)
\end{aligned}
$$

Since the term $Q\left(s_{-k}\right)$ is independent of the strategy of BS $k$, if BS $k$ changes the scheduled user from $q$ to $q^{\prime}$ we obtain:

$$
\begin{aligned}
& F\left(s_{k}, s_{-k}\right)-F\left(s_{k}^{\prime}, s_{-k}\right) \\
& =p_{q k}\left|\mathbf{v}_{k}^{T} \mathbf{h}_{q k}\right|^{2}-\sum_{b=1, b \neq k}^{B} p_{q b}\left|\mathbf{v}_{b}^{T} \mathbf{h}_{q b}\right|^{2} \\
& -\sum_{m=1, m \neq q}^{M} p_{m k}\left|\mathbf{v}_{k}^{T} \mathbf{h}_{m k}\right|^{2}-\beta \xi\left(I-I_{o}\right) \\
& -\left(p_{q^{\prime} k}\left|\mathbf{v}_{k}^{T} \mathbf{h}_{q^{\prime} k}\right|^{2}-\sum_{b=1, b \neq k}^{B} p_{q^{\prime} b}\left|\mathbf{v}_{b}^{T} \mathbf{h}_{q^{\prime} b}\right|^{2}\right. \\
& \left.-\sum_{m=1, m \neq q^{\prime}}^{M} p_{m k}\left|\mathbf{v}_{k}^{T} \mathbf{h}_{m k}\right|^{2}-\beta \xi\left(I^{\prime}-I_{o}\right)\right)
\end{aligned}
$$

that is equal to $U_{k}\left(s_{k}, s_{-k}\right)-U_{k}\left(s_{k}^{\prime}, s_{-k}\right)$.

For the MinInt algorithm the potential function can be easily set equal to its utility function leading directly to a potential game. Finally, the potential function for the LinComb algorithm is the sum of the two potential functions considered before and the proof is straightforward.

\section{REFERENCES}

[1] Cisco, "Cisco visual networking index: global mobile data traffic forecast update, 2013-2018,” Feb. 2014, White Paper, pp. 1-40.
[2] Pi Zhouyue and F. Khan, "An introduction to millimeter-wave mobile broadband systems," IEEE Comm. Mag., vol. 49, no. 6, pp. 101-107, June 2011.

[3] T. S. Rappaport, Shu Sun, R. Mayzus, Hang Zhao, Y. Azar, K. Wang, G. N. Wong, J. K. Schulz, M. Samimi and F. Gutierrez, "Millimeter wave mobile communications for 5G cellular: it will work!," IEEE Access, vol. 1, pp. 335-349, 2013.

[4] W. Roh, J. Y. Seol, J. Park, B. Lee, J. Lee, Y. Kim, J. Cho, K. Cheun and F. Aryanfar, "Millimeter-wave beamforming as an enabling technology for $5 \mathrm{G}$ cellular communications: theoretical feasibility and prototype results," IEEE Comm. Mag., pp. 106-113, Feb. 2014.

[5] T. Kim, J. Park, J. Seol, S. Jeong, J. Cho and W. Roh, "Tens of Gbps support with mmWave beamforming systems for next generation communications," IEEE GLOBECOM, pp. 3685-3690, 9-13 Dec. 2013.

[6] Metis project, "Intermediate description of the spectrum needs and usage principles," D5.1 Deliverable, Aug. 2013.

[7] CoRaSat project, "Service and market requirememts," D.2.1 Deliverable, Oct. 2013.

[8] ITU-R M.2109, "Sharing studies between IMT Advanced systems and geostationary satellite networks in the fixed-satellite service in the 34004200 and 4500-4800 MHz frequency bands," 2007.

[9] ITU-R 4-5-6-7/TEMP/139-E, "Sharing and compatibility between IMT system and fixed-satellite service network in $5850-6425 \mathrm{MHz}$ frequency range," Feb. 2007.

[10] D. Monderer and L. Shapley, "Potential games," Games and Economic Behavior, pp. 124-143, 1996.

[11] Nie Nie, C. Comaniciu, "Adaptive channel allocation spectrum etiquette for cognitive radio networks," J. Mobile Networks and Applications, vol. 11, no. 6, pp. 779-797, Dec. 2006.

[12] S. Buzzi, G. Colavolpe, D. Saturnino and A. Zappone, "Potential games for energy-efficient power control and subcarrier allocation in uplink multicell OFDMA systems," IEEE J. of Selected Topics in Signal Processing, vol. 6, no. 2, pp. 89-103, April 2012.

[13] Fu-Wen Chen and Jung-Chun Kao, "Game-based broadcast over reliable and unreliable wireless links in wireless multihop networks," IEEE Trans. on Mob. Comp., vol. 12, no. 8, pp. 1613-1624, Aug. 2013.

[14] F. Guidolin, M. Nekovee, L. Badia, M. Zorzi, "A study on the coexistence of fixed satellite service and cellular networks in a mmWave scenario," Proc. IEEE ICC 2015.

[15] T. Bai, V. Desai and R. W. Heath, "Millimeter wave cellular channel models for system evaluation," IEEE ICNC 2014, pp. 178-182, 3-6 Feb. 2014

[16] S. Hur, Y. J. Cho, T. Kim, J. Park, A. Molisch, K. Haneda and M. Peter "Wideband Spatial Channel Model in an Urban Cellular Environments At $28 \mathrm{GHz}$," Proc. EuCAP 2015, April 2015.

[17] A. Alkhateeb, O. El Ayach, G. Leus and R. W. Heath, "Hybrid precoding for millimeter wave cellular systems with partial channel knowledge," Proc. ITA Workshop, pp. 1-5, 10-15 Feb. 2013.

[18] IITU-R P.452-15, "Prediction procedure for the evaluation of interference between stations on the surface of the Earth at frequencies above about $0.1 \mathrm{GHz}$," Sept. 2013.

[19] ITU-R S.465, "Reference radiation pattern for earth station antennas in the fixed-satellite service for use in coordination and interference assessment in the frequency range from 2 to $31 \mathrm{GHz}$," Jan. 2010.

[20] ITU-R SF.558-2, "Maximum allowable values of interference from terrestrial radio links to systems in the fixed-satellite service employing 8-bit PCM encoded telephony and sharing the same frequency bands," July 1986.

[21] ITU-R Rec. S.1432, "Apportionment of the allowable error performance degradation to Fixed-Satellite Service (FSS) hypothetical reference digital paths arising from time invariant interference for system operating below $30 \mathrm{GHz}$," Jan. 2000.

[22] S. Rangan, T. S. Rappaport and E. Erkip, "Millimeter wave cellular wireless networks: potentials and challenges," Proc. of the IEEE, vol. 102, no. 3, pp. 366-385, March 2014. 